\title{
The application of waste management systems for long duration spaceflight
}

\author{
James M. Oglesby* \\ Institute for Simulation and Training, Department of Psychology, University of Central Florida, 3100 Technology \\ Parkway, Orlando, FL, 32826, United States of America
}

\begin{abstract}
In the future planned interplanetary expedition mission to Mars, spaceflight crewmembers will be exposed to an environment that is completely unique from anything they are accustomed to on Earth. Due to the characteristics of these missions, a challenge will be to design an environment that allows crewmembers to easily work and live in for extended durations. One of the challenges associated with these future missions is supplying the crew with essential resources for survivability such as food and water. In this case, the waste management system can play a role in a closed-loop life support system, as provisions sent with the crew will be severely limited with no opportunity for resupply. The following looks at the rationale of designing a system for collecting, storing, and recycling human bodily waste that (1) is considered user-friendly by crewmembers in regard to habitability in spaceflight, and (2) provides applications for a self sustaining closed-loop life support system that will aid the crew during the mission. Future design processes should consider adhering to these guidelines to help in the spaceflight crew's living environment and the conduction of the interplanetary expedition.
\end{abstract}

Keywords: aerospace, sustainability, spaceflight, waste management, resource management

\section{Introduction}

Research associated with spaceflight has evolved dynamically from the development of space programs to the advancement of technology currently applied to the domain. The questions developed by researchers of humans in space have also evolved, from if survivability in space is possible, to maintaining health and performance of crewmembers working in space [26]. Research associated with spaceflight must take into account a large number of elements associated with living and working in the isolated and confined area which enable crewmembers to live and work in a safe and productive manner.

In terms of living and working effectively in the environment crewmembers conduct their mission in, space habitability is important for its impact on performance and morale. Space habitability refers to the design, integration, and support of all the elements that apply to the spaceflight operational environment and considered to play a role impacting the individual [9]. This includes everything in the environment that the crewmembers are exposed to, such as communication devices, displays in the spacecraft, cloth- ing, food, hygiene facilities, etc. [13]. It is indicated that there are multiple approaches possible to improve space habitability through proper design of the crewmembers living and operational environment [9]. Examples of such approaches may consist of mitigation of ambient noise from sources to reduce stress, and the design of appliances used to optimize comfort of the spaceflight crew. In other words, design implementations of the environment are determined as potential countermeasures to meet the challenges of spaceflight similar to astronaut selection and training.

The consideration of space habitability will be a critical component in the future planned expeditions to Mars and the establishment of a habitable Martian colony for living. For these planned missions, one must take into account the unique demands associated with long duration spaceflight. With the consideration of these challenges, possible solutions can be developed in the spacecraft design and the space crew's interaction with these elements. These considerations can aid in the development of the spacecraft and the colony environment suitable for planetary exploration missions that ensures efficiency, sustain- 
ability, and survivability. One component in the spacecraft design is the system that collects and stores bodily waste during the spaceflight mission. The possible capabilities of the waste management system can not only promote efficient use and reliability, but also aid in supporting a self-sustaining life support system for the space exploration crew.

\section{Future considerations}

The following looks at two considerations in the design of a waste management system for the application in spaceflight missions that extend beyond the low orbit of Earth. First, the waste management system should consider a user-centered design that promotes convenience of use. Additionally, with the demands of an extended duration spaceflight and limited support from Earth, the waste management can act as a component of a closed-loop life support system for the crewmembers in the spacecraft.

\subsection{Ergonomics of the system.}

Due to conditions of microgravity, many procedures conducted on earth related to eating, drinking, and hygiene cannot be replicated completely in space. As gravity plays a large role in keeping food and liquids in containers, astronauts must take special measures to control consumable materials from interfering with daily routines in space $[1,25,27]$.

While current systems used by crewmembers may be considered adequate for its purpose of collecting and storing waste, and standards are in place for designing waste management system that are used as well as other appliances and devices [7]. However, even currently, issues still arise in the functionality and reliability of the waste management systems used in space, such as onboard the International Space Station [21]. The need of an effective and usable system is important for maintaining a habitable environment in space.

Examples regarding the importance of ergonomic principles can be seen from the use of waste bags during the National Aeronautics and Space Administration's Apollo space program. A report by McAllister [11] provides details of the system used to collect and store bodily waste matter during missions, which utilized a plastic bag for containing the waste. Crewmembers were required to expel the waste into the bag and then mix the content with germicide to avoid rupturing due to expanding gas. While this system for collecting waste was considered satisfactory from an engineering standpoint, individuals that were required to use it resented the process and the materials. Actions - such as changing their dietwere taken in order to avoid using the system. Two main reasons were found as the source of user dissatisfaction: First, the possibility of committing an error when using the system was high, with errors potentially resulting in unfavorable consequences (waste content escaping out of the bag). Second, crewmembers that used the system indicated a larger degree of manipulation for proper use compared to using waste units they were accustomed to on Earth. Succeeding implementations include the waste management system installed in Skylab consisting of a wall mounted fecal bag system [12]. In comparison to the Apollo waste management system, the wall mounted facility onboard Skylab would be considered more analogous to an appliance that crewmembers would use on Earth, which may be an approach to increase acceptance of the system.

A connection to crewmember's routines and habits is considered important to those that conduct the spaceflight mission. This preference of establishing a connection to Earth while in space plays a part back into the concept of space habitability. An example would be the dining room tables that are installed in the International Space Station (ISS). With the conditions of microgravity, the orientation of up (ceiling) and down (floor) is not as easily detected in a spaceflight environment as compared to on Earth. However, spaceflight crewmembers use the dining table in the orbital facility for eating and as a gathering area during missions, analogous to routines one is accustomed to on Earth. Also found in the ISS is the installation of lighting fixtures on only one side of the terminals. Specifically, with lights installed on one side of the terminal, one would interpret the side being the ceiling of the orbiting facility and the opposite side as the floor. The overall cues given in the environment provide a perception of up and down. Therefore, even though an individual has no vestibular sense of orientation, astronauts still behave according to the environmental cues of the lights and tables as if this orientation is present. Additionally, crewmembers show a preference for having these environmental cues in comparison to the more efficient approach of placing equipment on all of the walls of the facility sacrificing cues of vertical orientation $[10,27]$. With this guideline of designing according to what crewmembers are accustomed to can be transferred to developing a waste management 
system to contribute to the overall habitability of the spacecraft during long duration spaceflight missions.

Due to microgravity, the procedure of managing bodily waste is a challenging aspect during spaceflight. From a habitability perspective, the ideal system applied for spaceflight missions would reflect the characteristics of what the crewmembers are accustomed to on Earth. However, this is a difficult goal to achieve in terms of the system's functionality and reliability. Additionally, the design must also take into consideration the limited capacity in terms of size and weight of the spacecraft. Even with the uniqueness of the environment, user-centered designs can still be implemented for ease of use for the crewmembers.

The National Aeronautics and Space Administration (NASA) uses the Man-Systems Integration Standards [14] for developing the criteria for all of the tools and equipment used in space, focusing on functionality, reliability, and usability. This also pertains to the facilities that are applied for storing and managing waste in the spacecraft. Guidelines in the set standards focus on the system's reliability that minimizes its need for repair, functions well in microgravity, promotes ease of use, and is both physically and psychologically acceptable to the crewmembers. However, with the challenges that are considered to be faced in future missions to beyond the low orbit of Earth, additional guidelines may need to be in place that optimizes efficient use of resources.

\subsection{Sustainability for long duration spaceflight.}

Future system designs will need to take into account the challenges and requirements of extended durations in spaceflight compared to previously conducted low orbit missions. The mission to Mars is projected to be longer than any previous manned spaceflights [18], with a planned four to six months traveling to Mars, an 18-20 month stay on the Martian surface, and another four to six months traveling back to Earth [5]. Another distinction is the high level of autonomy that the crewmembers will be subjected to. Communication between the flight crew and Mission Control will be delayed up to 44 minutes due to the large distance to be traveled, eliminating the possibility of real time communication in cases of emergency [8]. Along with communication barriers, less reliance will be placed on support from outside of the spacecraft during the expedition to Mars. The use of supplies and resource is an upmost critical component as sending a rescue crew or extra supplies in case of an emergency will not be possible $[3,8,18$, 19].

With these demands, possibilities are considered for providing the spaceflight crew with the essential resources for survivability such as food and water. The first approach would be to send all of the resources needed with the launch of the spacecraft and requiring the crew to manage these finite set of resources effectively throughout the entire mission. From a financial standpoint, this approach is not entirely feasible, as the cost of launching one kilogram of weight into low orbit is approximately $\$ 11,000$, and this amount will increase for launches beyond low orbit [20]. Another approach to take in supplying the crew for the mission would be to develop a selfsustaining system that is capable of recycling waste materials into consumable resources. From both a practical and cost-effective standpoint, the capability of recycling materials and waste during the spaceflight mission is a consideration for interplanetary exploration [2]. Another approach to take would be a hybrid of the two previous approaches. In this case, there will be resources that are sent along with the crew for them to use, but they will also rely on a recycling system for providing a sustainable source of resources as well. This approach is considered the most likely approach to take for the future planned expedition to Mars [20].

In its most complex form, the life support system to be used for the mission would need to be capable of providing oxygen, water, food, and energy requirements during the expedition, being completely independent of outside support for obtaining these resources [6]. To accomplish this task, there needs to be regenerative capabilities for processing wastes into usable resources for humans. One approach for developing a closed-loop life support system is to focus on how waste material from the space crew can be utilized as a component for supplying consumable resources to sustain the crewmembers like food and water. An example of this approach is to include the implementation of plant life to supply these essential resources, as plants have been demonstrated to exhibit natural growth in microgravity environments [23]. Human waste can be an effective component in the harvesting of flora for supplying the crew with food and oxygen. Bodily wastes are a sufficient resource capable of providing plants with sufficient nutrients and water [15]. Possible psychological benefits on behalf of the crewmembers are also considered by implementing plant life [16]. Gardening tasks can be considered a recreational activity for crewmembers during the long flight in space, and 
many perceive the feasibility of the role of plant life as a solution for providing food.

The benefits of implementing recycling capabilities for spaceflight facilities can be seen in the installation of the urine treatment system that has been applied to the International Space Station (ISS). The use of this filtration system is capable of turning urine from the crewmembers into drinkable water completely free of any urine contaminants and pathogens for the astronauts onboard the orbiting facility. Several benefits are indicated with the use of this type of recycling system. First, with the overall cost of 250 million dollars for its development, the system is considered to be able to pay for itself and determine to be a more cost effective alternative to continuously send water to the station. Also, with the retirement of the Space Shuttle program and the limited cargo capacity of other spacecrafts traveling to the ISS, it would not be possible to send enough water to support a large crew of six members simultaneously in the facility [4].

\section{Conclusion}

The planning of future spaceflight missions involving interplanetary exploration presents challenges for both the crewmembers and the designers of the environment the crew will interact with. One of the factors to be considered is how bodily waste will be collected, stored, and used for recycling purposes in order to have less reliance on the limited supplies that would be shipped for the mission. Also, the role that space habitability plays into the morale of the crew should also be considered in the design of systems and appliances for spaceflight [17].

From an ergonomic standpoint, a practical implementation would be to design the system to reflect what the astronauts are accustomed to on Earth. However, exact duplicates of some appliances and facilities are not possible due to conditions of microgravity and other unique elements of the space environment. This also includes the challenges that need to be addressed in the development of a reliable and usable waste management system for spaceflight.

Urine and feces may not be necessarily labeled as waste if they have the capability to contribute to contribute to the functioning of a closed-loop life support system for the crew during the expedition. With the factors of future spaceflight exploration missions in hand, the planning of an effective waste management system can progress with a final product that is con- sidered satisfactory for the crew and contributes to maintaining the life support system during the mission. As artificially constructed closed-loop life support systems have been found to be able to provide a sustainable living and working environment for humans [22], focus needs to lead to practical applications of effective waste management systems for both the expedition to Mars, and for sustaining an established colony on the planet [19]. While the focus of this review is targeted towards the development and application of a sustainable waste management system for spaceflight, practical implications can potentially be applied to terrestrial settings. Developments and discoveries applied for spaceflight have successfully transferred to benefit society on Earth through technology, medicine, and research [24]. Effective applications of a waste management system in terms of resource management can be transferred to Earth for effective conservation of the finite resources for the inhabitants of Earth [28].

\section{References}

[1] M.M. Cohen (1998). Space habitat design integration issues, Proceedings of the $28^{\text {th }}$ International Conference on Environmental Systems, 1998.

[2] M. Czupalla, V. Aponte, S. Chappell and D., Analysis of spacecraft life support system for Mars mission, Acta Astronautica 55 (2004), 537-547.

[3] E. Gralla, S. Shull, M. Silver, J. Ahn, A. Siddiqi and O. De Weck, Remote terrestrial sites as operational/logistics analogs for Moon/Mars bases: The Haughton Mars Project, Paper presented at the SpaceOps 2006 Conference, 2006

[4] W. Harwood, Bottoms up: Space station crew samples recycled urine, retrieved from news.cnet.com/8301-19514_310245857-238.html, 2009.

[5] R. Hoffman and D. Kaplan, Human exploration of Mars: The reference mission of the NASA Mars exploration study team, 1997.

[6] C.J. Hurst, M.C. Roman, J.L. Garland, D.C. Obenhuber and A.M. Brittain, Microbiological aspects of space exploration, ASM News 63 (1997), 611-615.

[7] International Space Station Program, International Space Station flight crew integration standard (NASA-STD-3000/T). Retrieved from http://spacecraft.ssl.umd.edu/design_lib/ SSP50005rC.ISS_crew_integ.pdf, 1999.

[8] N. Kanas, Expedition to Mars: Psychological, interpersonal, and psychiatric issues, Journal of Cosmology 12 (2010), 37413747.

[9] N. Kanas and D. Manzey, Space Psychology and Psychiatry $2^{\text {nd }}$ edition, Springer, Netherlands, 2008.

[10] G.H. Kitmacher, Design of the Space Station habitable modules, Proceedings of the $53^{\text {rd }}$ International Astronautical Congress, 2002.

[11] F.A. McAllister, Apollo experience report- Crew provisions and equipment subsystem (NASA TN D-6737), National Aeronautical and Space Administration, Washington D.C., 1972. 
[12] S. Mohanty, M.J. Rycroft and M. Barratt (2001). Design concepts for zero-G whole body cleansing on ISS Alpha (NASA/CR-2001-208931), National Aeronautical and Space Administration, Houston TX, 2001.

[13] M.E. Morphew, Psychological and human factors in long duration spaceflight, McGill Journal of Medicine 6 (2001), 74-80.

[14] NASA (1995), Man-Systems Integration Standards, retrieved from http://msis.jsc.nasa.gov/default.htm.

[15] M., Nelson, A. Alling, W.F. Dempster, M. Van Thillo and J. Allen, Advantages of using subsurface flow constructed wetlands for wastewater treatment in space applications: Groundbased Mars base prototype, Advanced Space Research 31 (2003), 1799-1804.

[16] P. Santy, The journey out and in: Psychiatry and space exploration. The American Journal of Psychiatry 140 (1983), 519527.

[17] M.R. Schuh, A. Barr, J.H. Connolly, B. Woolford and M. Kaiser, Risk of reduced safety and efficiency due to inadequately designed vehicle, environment, tools, or equipment, retrieved from http://humanresearchroadmap.nasa.gov/evidence/reports-/inadequate\%20design.pdf, 2009.

[18] E. Seedhouse, Martian outpost: The challenges of establishing a human settlement on Mars. New York: Springer, 2009.

[19] S. Silverstone, M. Nelson, A. Alling and J. Allen, Development and research program for a soil-based bioregenerative agriculture system to feed a four person crew at a Mars base. Advanced Space Research 31 (2003), 69-75.
[20] R.C. Smith, Some biological considerations for a permanent, manned lunar base, The American Biology Teacher 54 (1995), 245-256.

[21] B. Stockman, J. Boyle and J. Bacon, International Space Station engineering case study, retrieved from http://handle.dtic.mil/100.2/ADA538763, 2010.

[22] V.N. Sychev, M.A. Levinskikh and I.G. Podolsky, Biological component of life support systems for a crew in longdurations space expeditions, Acta Astronautica 63 (2008), 1119-1125.

[23] V.N. Sychev, M.A. Levinskikh and Y.Y. Shepelev, The biological component of the life support system for a Martian expedition, Advanced Space Research, 31 (2003), 1693-1698.

[24] J. Vernikos, Human exploration of space: Why, where, what for?, Hippokratia 12 (2008), 6-9.

[25] H.A. Wichman, Behavioral and health implications of civilian spaceflight, Annual Review of Medicine, 54 (2005), 245 256.

[26] D.R. Williams, The biomedical challenges of spaceflight, Annual Review of Medicine, 54 (2003), 245-256.

[27] H.S. Wolff, Ergonomics in orbit, in: Contemporary Ergonomics, S.A. Robertson, ed., Taylor \& Francis Ltd, London, 1996, pp. 2-5.

[28] T. Wydeven and M.A. Golub, Waste streams in a crewed space habitat, Waste Management and Research 9 (1991), 91101. 